\title{
IFLCH: Intuitionistic Fuzzy Logic based Cluster Head Selection in Wireless Sensor Networks
}

\author{
A. Krishnakumar, D. Saraswathi
}

\begin{abstract}
A large number of tiny sensor nodes are grouped together to form Wireless Sensor Network (WSN). In Industry and other areas using of sensors are increasing every day. Therefore, the energy utilization of sensor nodes becomes a vital problem due to non-rechargeable battery. To improve the vital resources, the energy efficient clustering models are to be improved. This paper presents a novel idea IFLCH: Intuitionistic Fuzzy Logic based Cluster Head Selection for WSNs for electing Cluster Head $(\mathrm{CH})$ based on the energy efficiency parameters such as residual energy, distance between neighbors. The proposed scheme also elects Super $\mathrm{CH}$ (SCH) based on the above-mentioned parameters along with number of neighbors. The simulation results compared the proposed model with the existing schemes and it receives better performance by selecting efficient $\mathrm{CH}$ and $\mathrm{SCH}$.
\end{abstract}

Index Terms- Energy, Intuitionistic Fuzzy Logic, Sensor Nodes, Super Cluster Head (SCH), Number of Neighbors.

\section{INTRODUCTION}

Wireless Sensor Network (WSN) becomes a vital research area due to the technological improvement. This kind of network can be established anywhere without changing the atmosphere where it is to be deployed. Suppose, the traditional wired network needs to be wired and accessed in a certain point but the wireless network is easy to deploy without adding more hardware as like for wired networks. Generally, WSN consists a group of sensor nodes and a medium called Base Station (BS) that collects the sensed data from the sensor nodes. Mostly, the sensors deployed in hostile areas where the humans cannot easily access the environment. The sensor nodes access the signals like heat, light, etc., in general. The received signals will forwarded to the nearby BS where the received signals are stored and processed for other processes by the end user or humans.

As the deployment of sensor nodes are in hostile areas, the nodes are equipped with a fully charged nonrechargeable battery and processor. Mostly, the changing of batteries and recharging the batteries are not possible. Therefore, the need for energy-efficient communication model is to be identify and to improve lifetime of the network. One of the most commonly used protocol of WSN is Low Energy Adaptive Clustering Hierarchy (LEACH) by Heinzelman et al. [1, 2]. This protocol is one among the foremost protocol used to elect the $\mathrm{CH}$ for each round using its threshold function $T(n)$ in Eq. 1.

$$
\begin{aligned}
& T(n) \\
& 0, \frac{p}{\left(1-p \times\left(r \bmod \left(\frac{1}{p}\right)\right)\right)}, \text { if } n \in G
\end{aligned}
$$

Where $p$ denotes the probability of the nodes to become $\mathrm{CH}, r$ denotes the round which is ended, $G$ denotes the set of nodes which not been acted as $\mathrm{CH}$ in the last $1 / p$ rounds.

LEACH follows simple process for election of $\mathrm{CH}$. First, each node assigns with a numerical value between $0-1$ (Zero - One). Second, the assigned value checked with the threshold value that received through Eq.1. Third, the node that holds lesser value than the threshold value is act as $\mathrm{CH}$ for that round. The election of $\mathrm{CH}$ is a continuous process for each round. In this election, the node which holds a lesser energy, the node that is far away from BS, the node not maintain the centrality in a cluster than the other nodes of a cluster may become $\mathrm{CH}$. Therefore, the $\mathrm{CH}$ election is a serious effort making process in terms of improving the lifetime of the network.

The proposed scheme "IFLCH: Intuitionistic Fuzzy Logic based Cluster Head Selection for WSNs" follows the $\mathrm{CH}$ election based on the parameters node holding high residual energy and distance between neighbors. The proposed model also adds the election of Super $\mathrm{CH}(\mathrm{SCH})$ using Intuitionistic Fuzzy Logic (IFL). This scheme also proposes a dual power amplification model for $\mathrm{CH}$ and member nodes as well as for $\mathrm{SCH}$ and $\mathrm{CH}$.

The RELATED WORKS discusses the latest research work of different authors in Section II. The Section III tells about the IFEECH in detail and Section IV describes the EXPERIMENTAL SETUP discusses the analysis of the proposed model with the existing schemes. Finally, concludes with CONCLUSION in Section V.

\section{RELATED WORKS}

Designing of Energy-Efficient clustering protocols especially for utilising the low-energy for all functionalities of WSNs. Artificial Bee Colony (ABC) is one of among the best metaheuristic approach in optimizing the energy. The improved $\mathrm{ABC}$ (iABC) is the approach proposed by Mann \& Singh [3].

In this approach, the population approach is remodified to achieve the exploitation capabilities of the WSNs. the improved methodology follows the student's-t distribution, a new method to achieve the metaheuristic on WSNs. The proposed model utilizes minimal hardware utilities 


\section{IFLCH: INTUITIONISTIC FUZZY LOGIC BASED CLUSTER HEAD SELECTION IN WIRELESS SENSOR NETWORKS}

compared to the other existing approaches. The iABC elects the optimal $\mathrm{CHs}$ from the nodes based on the student's-t distribution value. Evaluating the performance of iABC using packet delivery, throughput, energy consumption and network lifetime. The design of $\mathrm{iABC}$ is suitable for small type of network and $\mathrm{i} A \mathrm{BC}$ detains its' performance for a large area networks.

Cognitive Improved LEACH (CogILEACH) is the model proposed by Rahman et al. [4]. The proposed methodology concentrates especially for the Cognitive Radio Sensor Networks (CRSN) [5]. The reliability is the major key for the CRSN. CogILEACH elects $\mathrm{CH}$ based on residual energy and number of neighbors. The ratio of the two metrics is calculated and the node that holds high ratio considered in acting as $\mathrm{CH}$. According to the need of end user, the assignment of weights to the each node is processed. At last, the modifications on the threshold function of LEACH with the parameters of CogILEACH obtains the proposed methodology.

Shokouhifar \& Jalali proposed optimized sugeno fuzzy clustering algorithm for WSN [6]. The model proposed adaptive fuzzy clustering protocol called LEACH-SF that consists of Fuzzy-C Means algorithm to balance cluster size. Then, achieves the election of $\mathrm{CH}$ using the threshold function of LEACH. Also generates the fuzzy rules based on the residual energy, distance to sink and node centrality in a cluster. Using ABC, the fuzzy rules are generated and adjusted. Thus, the proposed model achieves a better energy-efficiency than the existing schemes. The mobile sensor nodes, multi-hop routing are the further plans to implement with LEACH-SF model.

The model "A Fuzzy Logic based Clustering Algorithm for WSN to extend the Network Lifetime" introduces election of $\mathrm{SCH}$ based on fuzzy rules by Nayak \& Devulapalli [7]. The model elects $\mathrm{CH}$ based on the probabilistic threshold function of LEACH protocol. After the election, the model uses the Fuzzy Logic (FL) with the traditional mamdani rule fuzzy inference system to elect the $\mathrm{SCH}$ based on node using fuzzy descriptor remaining battery power, mobility of BS and centrality of the clusters. The proposed scheme verifies using some of the parameters such as First Node Dies (FND), Last Node Dies (LND), stability and lifetime. In addition, the sink considered as mobile in this scheme. The major flaw of the scheme is the election of $\mathrm{CH}$ is not determine the parameters to elect $\mathrm{SCH}$ node. Therefore, the node that holds a less energy may get a chance to elect as $\mathrm{SCH}$ that leads to degrade the lifetime of the network.

Prabhu et al. [8] proposed novel scheme to elect the $\mathrm{CH}$ based on the threshold value. The hierarchical distributed clustering algorithm LEACH is used with the modified threshold function. The parameters considered to elect the $\mathrm{CH}$ is based on the residual energy, distance and threshold energy. The threshold energy is possible to identify the minimal requirement of the node's energy to act as $\mathrm{CH}$ for a round. When a node satisfies the threshold energy then the node eligible to act as $\mathrm{CH}$ but still the nodes which satisfy the threshold energy will consider with the other parameters residual energy and distance to elect better node as $\mathrm{CH}$. The performance of the proposed scheme is compared with the LEACH.

\section{PROPOSED METHODOLOGY}

FL is used to develop a better decision making compared to the probabilistic approach. The member function of FL is used to identify the crisp set values. The IFL proposed in this model which is further useful to identify the member degree, non-member degree and hesitance value. Based on the member degree of a node with the modified fuzzy descriptor based on the proposed model the election of SCH is processed.

\section{Intuitionistic Fuzzy Logic}

IFL is one among the fuzzy set theory which is introduced by Atanassov [9]. The advantage of the scheme IFL is to deal with the vagueness [10]. The IFL model is also considered that the member function and non-member function values to be less than one but in FL member function value is only considered. The other functions like triangular, trapezoidal, etc. are common to the all two logics. The Intuitionistic Fuzzy Number is defined as in [10] for further understanding.

An IFN $\underset{A}{\sim i}$ is defined as:

i) An Intuitionistic Fuzzy Subset of the real line

ii) Normal, if any $\mathrm{x}_{0} \in \mathrm{R}$ such that $\mu_{A}^{\sim i}\left(\mathrm{x}_{0}\right)=$ 1 , then $\vartheta_{A}^{\sim i}\left(\mathrm{x}_{0}\right)=0$

iii) A Convex set for the membership function $\mu_{A}^{\sim i}(\mathrm{x})$ is

$\mu_{A}^{\sim i}\left(\lambda x_{1}+(1-\lambda) x_{2}\right) \geq \min \left(\mu_{A}^{\sim i}\left(x_{1}\right), \mu_{A}^{\sim i}\left(x_{2}\right),\right) \forall x_{1}, x_{2} \in R, \lambda \in[0,1]$

iv) A concave set for the non-membership function $\vartheta_{A^{\sim i}}$ is

$$
\vartheta_{A}^{\sim i}\left(\lambda x_{1}+(1-\lambda) x_{2}\right) \geq \min \left(\vartheta_{A}^{\sim i}\left(x_{1}\right), \vartheta_{A}^{\sim i}\left(x_{2}\right),\right) \forall x_{1}, x_{2} \in R, \lambda \in[0,1]
$$

The Triangular IF Number follows the Eq. 2 and Eq. 3 for membership function and non-membership function.

$$
\mu_{A}^{\sim i}(x)= \begin{cases}\frac{x-a_{1}}{a_{2}-a_{1}} \text { for } a_{1} \leq x \leq a_{2} \\ \frac{a_{3}-x}{a_{3}-a_{2}} \text { for } a_{2} \leq x \leq a_{3} \\ 0 \quad \text { otherwise }\end{cases}
$$


TABLE I

MEMBERSHIP VARIABLES WITH DEGREE (INPUT)

\section{MEMBERSHIP FUNCTIONS}

\begin{tabular}{ccc}
\hline \hline Residual Energy & $\begin{array}{c}\text { Distance between } \\
\text { Neighbors }\end{array}$ & Number of Neighbors \\
\hline LOW (0) & FARAWAY (0) & LESS (0) \\
MEDIUM (1) & NEARBY (1) & MINIMUM (1) \\
HIGH (2) & CLOSE (2) & VAST (2)
\end{tabular}

The above table shows the membership functions as an input to the criteria specified.

$$
\vartheta_{A}^{\sim i}(x)= \begin{cases}\frac{a_{2}-x}{a_{2}-a_{1}^{\prime}} \text { for } a_{1}^{\prime} \leq x \leq a_{2} \\ \frac{x-a_{2}}{a_{3}^{\prime}-a_{2}} \text { for } a_{2} \leq x \leq a_{3}^{\prime} \\ 1 \quad \text { otherwise }\end{cases}
$$

where $a_{1}^{\prime} \leq a_{1} \leq a_{2} \leq a_{3} \leq a_{3}^{\prime}$

Based on the above Eq. 2 and Eq. 3 the membership value and non-membership value will be obtained for the proposed scheme.

\section{Energy Model Analysis}

The amount of energy consumed for $l$ bits to a distance $d$ is as follows in Eq. 4.

$$
\begin{gathered}
E_{T x}(l, d)=E_{T x}-\operatorname{amp}(l, d) \\
=\left\{\begin{array}{l}
l * E_{\text {elec }}+l * \varepsilon_{f s} * d^{2} \text { if } d<d_{0} \\
l * E_{\text {elec }}+l * \varepsilon_{m p} * d^{4} \text { if } d \geq d_{0}
\end{array}\right.
\end{gathered}
$$

Where $E_{\text {elec }}$ denotes the dissipated energy to execute a bit of information. The energy may use to activate transmitter or receiver circuit depends on the function. Here, the functions denote spreading, filtering, modulation of a signal, $\varepsilon_{f s}$ and $\varepsilon_{m p}$ denotes the energy co-efficient values. The energy spent to receiving data is defined using Eq. 5.

$$
E_{R x}(l)=E_{R x}-\operatorname{elec}(l)=l * E_{\text {elec }}
$$

The distance between node $i$ and neigh $(i)$ is $d(i$, neigh $(i))$ calculated using Eq. 6.

$$
d(i, \operatorname{neigh}(i)) \in\left(X, \sqrt{(H / X)^{2}+(X / W)^{2}}\right)
$$

\section{System Model}

The system model emphasis election of $\mathrm{CH}$ using the two parameters: high residual energy, distance between neighbors.

The system follows the election of $\mathrm{CH}$ for the round 1 as it is in LEACH Protocol. The model is proposed from the round two to elect the $\mathrm{CH}$ using the above said parameters. To obtain residual energy of a node, the Eq. 7 is used.

$$
\operatorname{Rem}_{\text {egy }}=\frac{E_{\text {current }}}{E_{\text {maximum }}}
$$

where the $R e m_{\text {egy }}$ denotes the remaining energy of node, $E_{\text {current }}$ denotes the current energy value of a node and $E_{\text {maximum }}$ denotes a maximum energy which is an initial energy of a node.

The distance between neighbor nodes is obtained using Eq. 8.

$$
\operatorname{dist}_{n e i g h}=1-\left[\frac{\sum_{i=1}^{N} d i s_{i}}{N X d i s_{\max }}\right]
$$

where the dist $t_{\text {neigh }}$ denotes the distance between the neighbors, $d i s_{i}$ denotes the distance of node $i$ and $d i s_{\max }$ denotes the maximum distance of a node and $N$ denotes the number of neighbor nodes.

Both the Eq. 7 and Eq. 8 is added with the Eq. 1 forms the new model using Eq. 9.

$$
\begin{aligned}
& T(n)=\left[\left[\frac{E_{\text {current }}}{E_{\text {maximum }}}\right]+\left[1-\left[\frac{\sum_{i=1}^{N}}{N X \text { dis }}\right.\right.\right. \\
& \times\left\{\frac{p}{\left(1-p \times\left(r \bmod \left(\frac{1}{p}\right)\right)\right)}, \text { if } n \in G\right. \\
& 0, \quad \text { otherwise }
\end{aligned}
$$

Thus, the election of $\mathrm{CH}$ is processed as of the new model in Eq. 9 to obtain better $\mathrm{CH}$ which holds higher residual energy and higher number of neighbors. After the completion of election of $\mathrm{CH}$, the model concentrates in election of $\mathrm{SCH}$. The $\mathrm{SCH}$ election is processed using the IFL. Except the identification of membership and nonmembership values the IFL follows the same FL utilities.

\section{Intuitionistic Fuzzy Logic Model}

A Fuzzifier, Fuzzy Inference Engine, Fuzzy Rules and Defuzzifier are the common modules of FL. The famous Mamdani method [11] is used in this scheme which is commonly used in FL. The major four steps for any FL is fuzzification, rule evaluation, fuzzy inference engine and defuzzification.

The IFL for the proposed methodology use three fuzzy input to elect the SCH. The inputs are, residual energy, Number of neighbors and distance between neighbors. The two parameters residual energy and distance between neighbors are calculates as of in Eq. 7 and Eq. 8. The number of neighbors of a node will be calculated as of in Eq. 10.

$$
\text { Num }_{\text {neigh }}=\left[1-\frac{\left(\text { cnt }_{\text {neigh }}-\text { threshold }^{2}\right)}{\text { threshold }^{2}}\right]
$$

where $\mathrm{Num}_{\text {neigh }}$ denotes the number of neighbors of a node, $c n t_{\text {neigh }}$ denotes the neighbors of a current node and threshold denotes the threshold value of a node.

The threshold value for Eq. 10 is shown in Eq. 11. 


\section{IFLCH: INTUITIONISTIC FUZZY LOGIC BASED CLUSTER HEAD SELECTION IN WIRELESS SENSOR NETWORKS}

threshold $=\frac{N_{\text {total }}}{K_{\text {opt }}}$

where $N_{\text {total }}$ denotes the total number of nodes and $K_{\text {opt }}$ denotes the optimal number of clusters. After the selection of input variables, the membership functions can be framed. The membership functions and its degree for the proposed model is shown in Table I.

Table II shows the output membership functions with degree values.

The rule evaluation model evaluates the input functions in Table 1 with the IF...THEN... ELSE rules and it receives any one output membership functions from the Table II. The proposed model used 27 rules to evaluate. The rules with the fuzzy chance value is processed using the Eq. 12.

Fuzzy Chance $=$ Residual Energy + Distance between neighbors+ Number of Neighbors (12) III.

The evaluated results using the Eq. 12 is shown in Table

After the completion of election of $\mathrm{SCH}$, the $\mathrm{SCH}$ asks all the $\mathrm{CH}$ to forwards the data to it. The $\mathrm{SCH}$ node aggregates the information and forwards it to BS. By this model, the BS receives data from a single node which eradicate the collision at BS completely.

TABLE II

MEMBERSHIP VARIABLES WITH DEGREE (OUTPUT)

\section{MEMBERSHIP FUNCTIONS}

Very Weak (0), Weak (1), Low Adequate (2), Adequate (3), High Adequate (4), durable (5), High durable (6).

The above table shows the membership functions as an output to the criteria specified.

TABLE III

FUZZY RULES EVALUATION WITH FUZZY CHANCE MEMBERSHIP FUNCTIONS WITH FUZZY CHANCE

\begin{tabular}{clll}
\hline \hline $\begin{array}{c}\text { Residual } \\
\text { Energy }\end{array}$ & $\begin{array}{c}\text { Distance } \\
\text { between } \\
\text { Neighbors }\end{array}$ & $\begin{array}{c}\text { Number of } \\
\text { Neighbors }\end{array}$ & \multicolumn{1}{c}{$\begin{array}{c}\text { Fuzzy } \\
\text { Chance }\end{array}$} \\
\hline LOW (0) & FARAWAY (0) & LESS (0) & Very Weak (0) \\
LOW (0) & FARAWAY (0) & MINIMUM (1) & Weak (1) \\
LOW (0) & FARAWAY (0) & VAST (2) & Low Adequate (2) \\
LOW (0) & NEARBY (1) & LESS (0) & Weak (1) \\
LOW (0) & NEARBY (1) & MINIMUM (1) & Low Adequate (2) \\
LOW (0) & NEARBY (1) & VAST (2) & Adequate (3) \\
LOW (0) & CLOSE (2) & LESS (0) & Low Adequate (2) \\
LOW (0) & CLOSE (2) & MINIMUM (1) & Adequate (3) \\
LOW (0) & CLOSE (2) & VAST (2) & High Adequate (4)
\end{tabular}

TABLE III

FUZZY RULES EVALUATION WITH FUZZY CHANCE

\begin{tabular}{|c|c|c|c|}
\hline \multicolumn{3}{|c|}{$\begin{array}{c}\text { MEMBERSHIP FUNCTIONS WITH } \\
\text { FUZZY CHANCE }\end{array}$} & \multirow[b]{2}{*}{$\begin{array}{c}\text { Fuzzy } \\
\text { Chance }\end{array}$} \\
\hline $\begin{array}{l}\text { Residual } \\
\text { Energy }\end{array}$ & $\begin{array}{c}\text { Distance } \\
\text { between } \\
\text { Neighbors }\end{array}$ & $\begin{array}{l}\text { Number of } \\
\text { Neighbors }\end{array}$ & \\
\hline Medium (1) & Faraway (0) & Less $(0)$ & Weak (1) \\
\hline Medium (1) & Faraway (0) & Minimum (1) & Low Adequate (2) \\
\hline Medium (1) & Faraway (0) & Vast (2) & Weak (1) \\
\hline Medium (1) & Nearby (1) & Less $(0)$ & Low Adequate (2) \\
\hline Medium (1) & Nearby (1) & Minimum (1) & Adequate (3) \\
\hline Medium (1) & Nearby (1) & Vast (2) & Low Adequate (2) \\
\hline Medium (1) & Close (2) & Less $(0)$ & Adequate (3) \\
\hline Medium (1) & Close (2) & Minimum (1) & High Adequate (4) \\
\hline Medium (1) & Close (2) & Vast (2) & Durable (5) \\
\hline High (2) & Faraway (0) & Less $(0)$ & Low Adequate (2) \\
\hline High (2) & Faraway (0) & Minimum (1) & Weak (1) \\
\hline High (2) & Faraway (0) & Vast (2) & Low Adequate (2) \\
\hline High (2) & Nearby (1) & Less $(0)$ & Adequate (3) \\
\hline High (2) & Nearby (1) & Minimum (1) & Low Adequate (2) \\
\hline High (2) & Nearby (1) & Vast (2) & Adequate (3) \\
\hline High (2) & Close (2) & Less $(0)$ & High Adequate (4) \\
\hline High (2) & Close (2) & Minimum (1) & Durable (5) \\
\hline High (2) & Close (2) & Vast (2) & High Durable (6) \\
\hline
\end{tabular}

The above table shows the membership functions with the fuzzy chance.

\section{EXPERIMENTAL RESULTS}

To evaluate the proposed scheme, the model is simulated using NS-2 [12,13] simulator (2.35) to compare the performance with the $\mathrm{LEACH}$ and $\mathrm{LEACH}$ with FUZZY

TABLE IV

\begin{tabular}{ll}
\hline \hline \multicolumn{1}{c}{ PARAMETER } & \multicolumn{1}{c}{ VALUE } \\
\hline Network Size & $100 \times 100 \mathrm{M}$ \\
Number of Nodes & $100(0-99)$ \\
BS Node & 100 \\
BS location & 50,175 \\
Initial Energy & $1 \mathrm{~J}$ \\
E $_{\text {elec }}$ & $25 \mathrm{~nJ} / \mathrm{bit}$ \\
$\varepsilon_{f s}$ & $5 \mathrm{pJ} / \mathrm{bit} / \mathrm{m}^{2}$ \\
$\varepsilon_{m p}$ & $0.0008 \mathrm{pJ} / \mathrm{bit}^{2} / \mathrm{m}^{4}$ \\
Simulation Time & $20000 \mathrm{~s}$ \\
Round Time & $20 \mathrm{~s}$
\end{tabular}

The Simulation parameters are specified as of in the above table to simulate the using NS-2 simulator.

Descriptor schemes with parameters such as residual energy, number of alive nodes, First Node Dies (FND) and so on. The simulation parameters are given in Table 4 .

Figure 1 shows the average residual energy of node. The residual energy shows the lifetime of a node.

From the Figure 1. the proposed model holds the high residual energy than LEACH with fuzzy descriptor and LEACH. 
Figure 2. shows the number of alive nodes among the schemes. The number of alive nodes improves the lifetime and also the scalability of the network.

The proposed model achieves high number of alive nodes compared to the existing models and thus proves the model is energy efficient.

Figure 3. shows the FND between the proposed and existing schemes where the effectiveness of the proposed model to be proved.

The above figures proves the proposed model is high effective compared to the existing models and it improves the network lifetime.

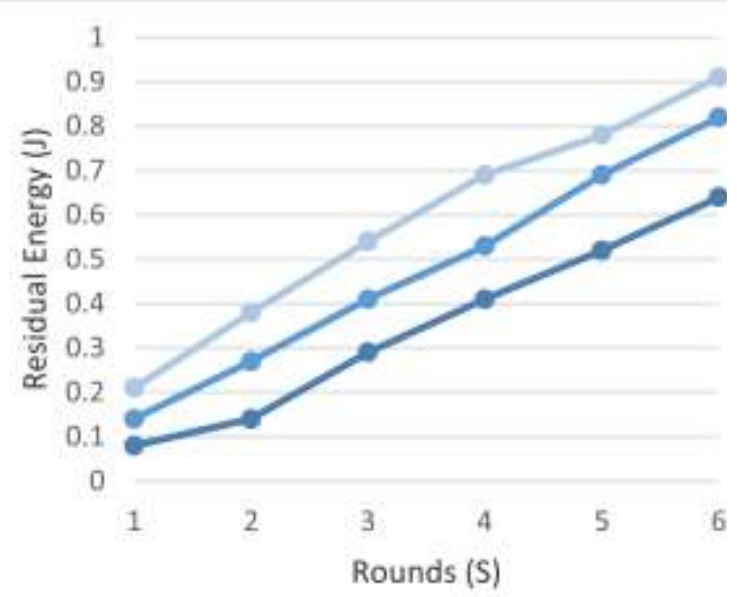

$\rightarrow$ LEACH $\rightarrow$ LEACH with FUZZY $\rightarrow$ IFLCH

Fig. 1. Compares the proposed model with the existing schemes and proposed model holds higher residual energy than the existing schemes.
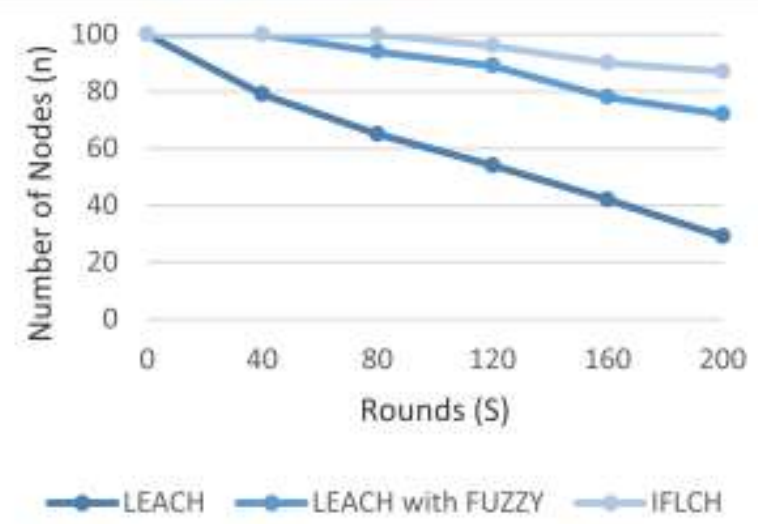

Fig. 2. Compares the proposed model with the existing schemes and proposed model holds higher number of alive nodes than the existing models.

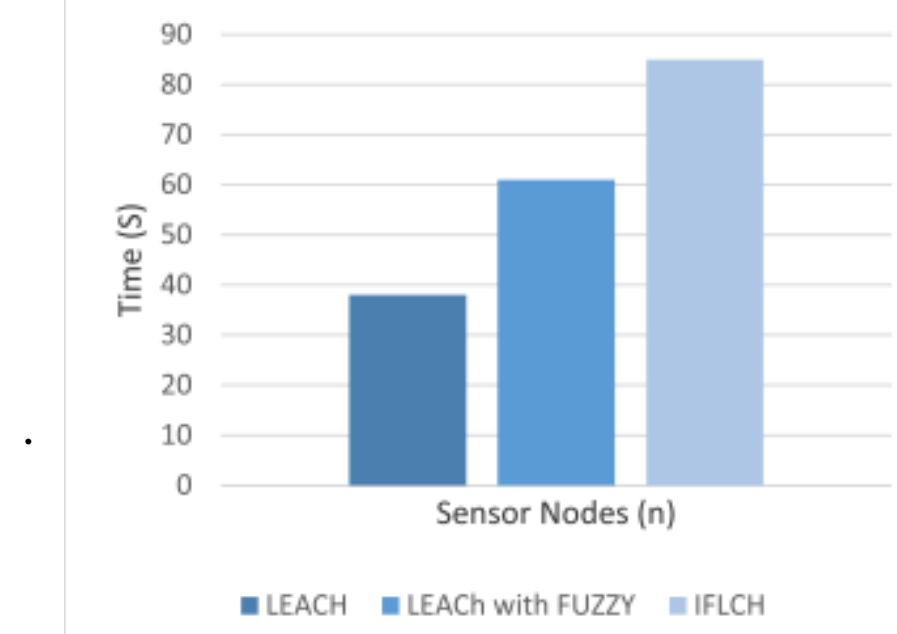

Fig. 3. Compares the proposed model with the existing schemes and proposed model holds more time for the occurrence of FND (First Node Dies).

\section{V.CONCLUSION}

The Proposed model is focus on improving the effective selection of $\mathrm{CH}$ with the modified threshold function of LEACH Protocol using residual energy and number of neighbors. The residual energy concentrates reducing energy consumption and distance between neighbors concentrates in the centrality of a node. By combining these two parameters, the threshold function of LEACH becomes efficient in election of $\mathrm{CH}$. after the completion of $\mathrm{CH}$ election process the proposed model concentrates in election of $\mathrm{SCH}$ using three parameters residual energy, number of neighbors and distance between neighbors. To do this, the Intuitionistic Fuzzy Logic is used. The $\mathrm{CH}$ nodes are processed with the fuzzy input and receives the fuzzy chance. The $\mathrm{CH}$ node that holds higher fuzzy chance value is elected as $\mathrm{SCH}$ for that round. By communicating a single node to $\mathrm{BS}$, the collision is totally avoided. The simulation results prove that the proposed model performs better than the existing schemes in all aspects. The parameters to elect $\mathrm{CH}$ and $\mathrm{SCH}$ can be defined with this scheme for future developments.

\section{REFERENCES}

1. Heinzelman, W. R., Chandrakasan, A., \& Balakrishnan, H. (2000, January). Energy-efficient communication protocol for wireless microsensor networks. In System sciences, 2000. Proceedings of the 33rd annual Hawaii international conference on (pp. 10-pp). IEEE.

2. Heinzelman, W. B., Chandrakasan, A. P., \& Balakrishnan, H. (2002). An application-specific protocol architecture for wireless microsensor networks. IEEE Transactions on wireless communications, 1(4), 660-670.

3. Mann, P. S., \& Singh, S. (2017). Improved metaheuristic based energy-efficient clustering protocol for wireless sensor networks. Engineering Applications of Artificial Intelligence, 57, 142-152.

4. Rahman, M. M., Miah, M. S., \& Sharmin, D. (2017). Cognitive Improved LEACH (CogILEACH) Protocol for Wireless Sensor 
Network. Transactions on Networks and Communications, 4(6), 01.

5. KLA Yau, P Komisarczuk, PD Teal, in IEEE 34th Conference on Local Computer Networks. Cognitive radio-based wireless sensor networks: conceptual design and open issues, (2009), pp. 955-962.

6. Shokouhifar, M., \& Jalali, A. (2017). Optimized sugeno fuzzy clustering algorithm for wireless sensor networks. Engineering Applications of Artificial Intelligence, 60, 16-25.

7. Nayak, P., \& Devulapalli, A. (2016). A fuzzy logic-based clustering algorithm for wsn to extend the network lifetime. IEEE sensors journal, 16(1), 137-144.

8. Prabhu, B., Balakumar, N., \& Antony, A. J. (2017). A Novel LEACH Based Protocol for Distributed Wireless Sensor Network.

9. Atanassov, K. T. (1986). Intuitionistic fuzzy sets. Fuzzy sets and Systems, 20(1), 87-96.

10. Mahapatra, G. S., \& Roy, T. K. (2013). Intuitionistic fuzzy number and its arithmetic operation with application on system failure. Journal of uncertain systems, 7(2), 92-107.

11. Mamdani, E. H. (1976). Advances in the linguistic synthesis of fuzzy controllers. International Journal of Man-Machine Studies, 8(6), 669-678.

12. Wei, D., Jin, Y., Vural, S., Moessner, K., \& Tafazolli, R. (2011). An energy-efficient clustering solution for wireless sensor networks. IEEE transactions on wireless communications, 10(11), 3973-3983.

13. Singh, B., \& Lobiyal, D. K. (2012). An energy-efficient adaptive clustering algorithm with load balancing for wireless sensor network. International Journal of Sensor Networks, 12(1), 37-52.

\section{AUTHORS PROFILE}

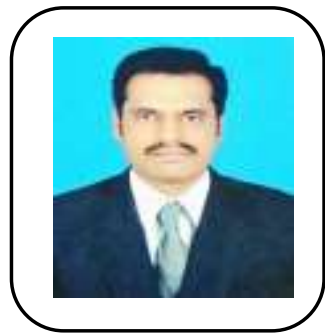

Dr. A.Krishnakumar has 6 years of teaching experience. He has completed his Master degree in Computer Science at Bharathiar University, Coimbatore. He did his M.Phil at Karpagam University \& Ph.D (Computer Science) at Bharathiar University,Coimbatore.

Prof. Krishnakumar has published papers in IEEE Xplore, ESCI Index, Scopus and Google Scholar. He has published 5 research papers in International Journals. He has published 8 Papers in various National and International Conferences. He also guided numerous PG and UG students for their projects. She is a member of Institute of Electrical and Electronic Engineers (IEEE), and IFERP. His area of specialization is Wireless Sensor Networks.

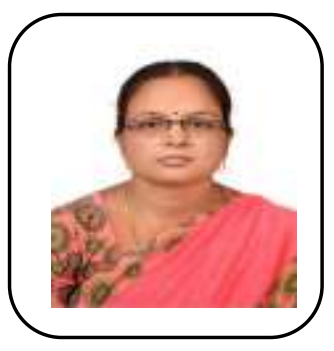

Dr. D. Saraswathi has 12 years of teaching experience. She has completed her Master degree in Software Science (5 years integrated) at Periyar University, Salem. She did her M.Phil \& Ph.D (Computer Science) at Periyar University, Salem.

Prof. Saraswathi has published papers in Elsevier, IEEE Xplore, SCI Index, Scopus, Google Scholar and Springer. Her Google scholar citations are 36. She has published 14 research papers in International Journals. She has published 34 Papers in various National and International Conferences. She has attended 26 workshops and Faculty Development Programme. She also guided numerous PG and UG students for their projects. She is a member of International Association of Engineers (IAENG), and ICTACT. Her area of specialization are web mining and text mining. 